\title{
Generating Nanodot Structures on Stainless-Steel Surfaces by Cross Scanning of a Picosecond Pulsed Laser
}

\author{
Tomoki Kobayashi ${ }^{1} \cdot$ Jiwang Yan ${ }^{1}$
}

Received: 4 February 2020 / Revised: 5 April 2020 / Accepted: 10 April 2020 / Published online: 30 April 2020

(c) The Author(s) 2020

\begin{abstract}
Ultrashort pulsed laser-induced periodic surface structures (LIPSS) can be generated on different kinds of materials, which are widely utilized for modifying surface properties such as wettability, adhesion, and tribological, as well as optical performances. Previous studies have focused mainly on one-dimensional LIPSS (i.e., line structure) generation. In this study, a picosecond pulsed laser was used to irradiate stainless-steel surfaces for generating two-dimensional LIPSS, namely nanodot structures, by cross-scanning the laser beam for a different number of times. The obtained nanodot structures were found to be super hydrophilic just after laser irradiation, but turned to be hydrophobic after exposure in air for a few days. By crossscanning the laser beam for the same number of times, local LIPSS rewriting was realized. This study showed the possibility of improving the homogeneity of the surface properties of steel materials through laser-induced nanodot structuring.
\end{abstract}

Keywords Nanodot structure $\cdot$ Surface patterning $\cdot$ Picosecond pulsed laser $\cdot$ LIPSS $\cdot$ Tool steel $\cdot$ Wettability control

\section{Introduction}

Formation of laser-induced periodic surface structures (LIPSS) has been confirmed on various kinds of materials such as metals, semiconductors, and dielectrics by irradiating an ultrashort pulse laser near the ablation threshold [1-6]. The period of LIPSS is shorter than the incident laser wavelength [7], and the direction of LIPSS is determined by the laser polarization [8-10]. One of the possible LIPSS formation mechanisms is the interference of laser and the surface plasmon polariton (SPP) excited by the laser [11, 12]. An irradiating laser generates an electric charge distribution on the surface and induces SPP. The laser-SPP interference modulates the energy deposition. As a result, LIPSS is formed through ablation under the modulated energy deposition. Another possible mechanism is laser-induced material self-organization [5, 13]. After electrons are emitted from a surface during laser irradiation, the surface will become unstable and self-organization occurs. As a result, LIPSS is assembled via surface relaxation. The main mechanism for

Jiwang Yan

yan@mech.keio.ac.jp

1 Department of Mechanical Engineering, Faculty of Science and Technology, Keio University, Hiyoshi 3-14-1 Kohoku, Yokohama, Kanagawa 223-8522, Japan
LIPSS formation on a specific material depends on its material property and the atmosphere or media in which laser irradiation is done [2,14].

Although the physics of LIPSS formation is still a matter of controversy, LIPSS has been utilized by many researchers to alter surface wettability $[15,16]$, tribological property [17], optical performances [18, 19], and mold-releasing ability in plastic forming [2]. It is reported that laser wavelength [7, 20], polarization [8], fluence [11], number of pulses [21-23], incident angle [24, 25], and pulse repetition [26] affect the period of LIPSS. Furthermore, the morphology of LIPSS is dependent on the surrounding media [14]. However, to date, only one-dimensional LIPSS (parallel line structure) has been generated and there is little literature on two- or three-dimensional LIPSS formation.

One-dimensional LIPSS is highly directional, and thus may cause anisotropy in the surface property. For example, in plastic molding applications [2], line-structure LIPSS may cause nonhomogeneous plastic flow near the surface of the mold, and in turn, lead to residual stresses and even form errors on the molded components. From this meaning, twodimensional LIPSS or locally modified surface line-structure becomes necessary, which is helpful in improving the uniformity of interfacial plastic flows.

In this study, we aim at generating two-dimensional LIPSS, namely nanodot structures, and other novel surface 
patterns on stainless-steel surfaces by picosecond pulsed laser irradiation. The laser is irradiated on the workpiece surface for a certain number of times by crossing the beam scan directions perpendicularly. The historical effect of LIPSS formation is investigated under various conditions. The wettability change of the resulting surfaces is evaluated to verify the effectiveness of the proposed method in surface functionalization.

\section{Experimental Method}

In this study, a picosecond fiber laser PFLA-1030TP (Optoquest, Japan) was used for experiments. The configuration of the experimental system is shown in Fig. 1. The laser beam passes through a multi-frequency acousto-optic modulator (AOM), an attenuator and a beam expander, and then reflected by a mirror toward the sample surface. An objective lens was used to focus the laser beam, and a CCD camera was equipped for laser spot observation. A three-axis stage was used to move the workpiece in different directions.

The laser wavelength was $1030 \mathrm{~nm}$ and the pulse width was $50 \mathrm{ps}$ at a pulse frequency of $100 \mathrm{kHz}$. The laser beam was Gaussian and the spot size was $6-7 \mu \mathrm{m}$ with a focal length of $20 \mathrm{~mm}$. No defocus was used in this study. Fluence was set to 0.10 and $0.15 \mathrm{~J} / \mathrm{cm}^{2}$ in the experiments. The scanning speed was changed in the range of $1-40 \mathrm{~mm} / \mathrm{s}$. The scan overlap was set to 2 and $6 \mu \mathrm{m}$, respectively. The number of scans $(N)$, which was defined as the number that the laser was irradiated on the same area, was changed in the range of $1-100$.

Figure 2 shows a schematic of cross-scanning pattern of the laser beam. First, the laser was irradiated by scanning the beam along the horizontal direction in Fig. 2 (indicated as first irradiation) for a number of times $\left(N_{1}\right)$. After that, the workpiece was rotated $90^{\circ}$, and the laser was irradiated according to the same scheme (indicated as second irradiation) for another number of times $\left(N_{2}\right)$ to investigate how the

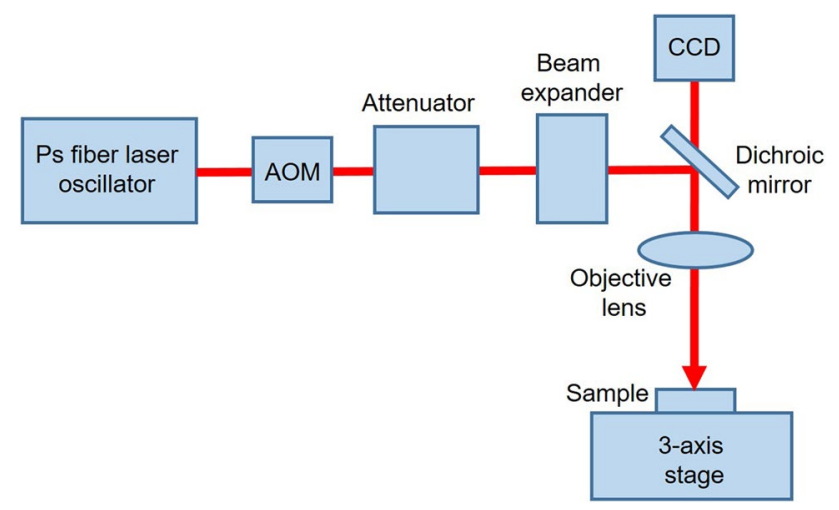

Fig. 1 Schematic of the experimental setup

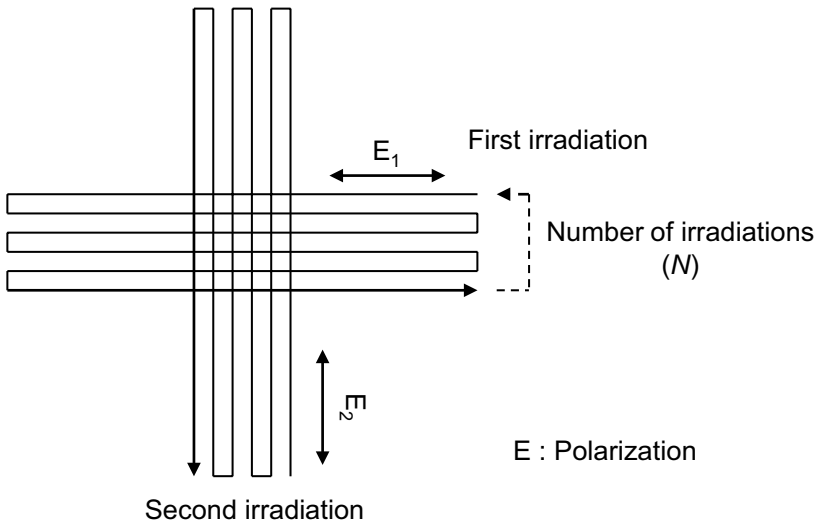

Fig. 2 Schematic of cross-scanning pattern of laser beam

two irradiations affect each other. The polarization of the laser beam for the two scan directions is $\mathrm{E}_{1}$ and $\mathrm{E}_{2}$, respectively, as indicated in the figure.

Stainless steel was used as the workpiece material. The mass percent of each material composition is $\mathrm{C}: 0.38 \%, \mathrm{Si}$ : $0.9 \%, \mathrm{Mn}: 0.5 \%, \mathrm{Cr}: 13.6 \%$, and V: $0.3 \%$. A scanning electron microscope (SEM) (INSPECT S50 produced by FEI Company, USA) was used to observe the obtained surface structures. An atomic force microscope (AFM) SPM-3 (Hitachi, Japan) was used to measure the profile of the LIPSS structure. A laser microscope, VK-9700 produced by KEYENCE CORPORATION, Japan, was used to measure the surface roughness of the samples.

After the irradiating laser, the wettability of the surface was investigated. The contact angle of water was measured after cleaning the sample with ethanol. Then, the sample was kept in the air for 7 days, and the contact angle was measured again. The droplet of water on the sample was observed with a contact angle meter, Simage Entry 5 produced by Excimer Inc., Japan.

\section{Results and Discussion}

\subsection{Effect of Repetitive Irradiation on Depth of LIPSS}

To examine the effect of repetitive irradiation on the depth change of LIPSS, the laser was irradiated along the same direction by changing the number of scans $(N)$ from 1 to 100. Figure 3 presents SEM images of the surfaces irradiated at a fluence of $0.10 \mathrm{~J} / \mathrm{cm}^{2}$ and scanning speed of $40 \mathrm{~mm} / \mathrm{s}$ by changing $N$ are shown in. When $N=1$ (Fig. 3a), fine nanoscale line structure parallel to the laser polarization is observed. This nanostructure is high spatial frequency LIPSS (HSFL) with a period considerably shorter than the laser wavelength [25]. When $N=3$ (Fig. 3b), in addition to 

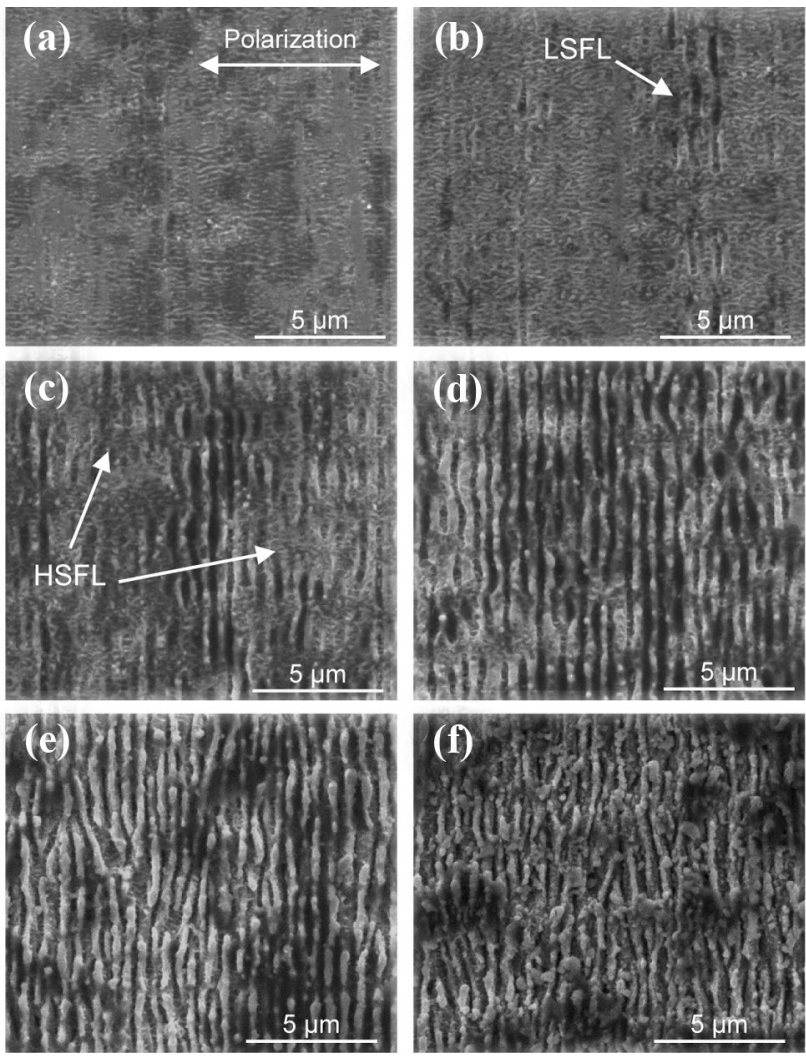

Fig. 3 SEM images of surfaces irradiated at fluence of $0.10 \mathrm{~J} / \mathrm{cm}^{2}$ and $N$ of a 1, b 3, c 5, d 10, e 50, f 100

HSFL, a few fine grooves perpendicular to the laser polarization are observed, which is presumably the low spatial frequency LIPSS (LSFL) [27]. At $N=5$ (Fig. 3c), the formation of LSFL becomes dominant in most of the area, while the area with HSFL formation is decreased. When $N=10,50$, and 100 (Fig. 3d-f), LSFL is formed on the entire surface without HSFL.

Normally, HSFL is formed at lower fluence than LSFL [25]. In this study, it is demonstrated that even at low fluence, a transition from HSFL to LSFL occurs by increasing the number of scans $N$. This transition maybe caused by surface roughening. The irradiated surface becomes rougher by the formation of HSFL, thus the absorption rate of light is increased [10]. Therefore, the energy absorbed in the surface is increased, and LSFL is formed by repetitive irradiations.

The SEM images of the surfaces irradiated at higher fluence, $0.15 \mathrm{~J} / \mathrm{cm}^{2}$, and the same scanning speed $(40 \mathrm{~mm} / \mathrm{s})$ with changing the number of scans $N$ are shown in Fig. 4. Unlike the case of $0.10 \mathrm{~J} / \mathrm{cm}^{2}$, HSFL is not observed in Fig. 4. In contrast, LSFL is formed on all the surfaces, even at $N=1$. As $N$ increases, the depth of the LIPSS also increases. This result indicates that it is necessary to use sufficiently high laser fluence to obtain LSFL by a single laser scan.
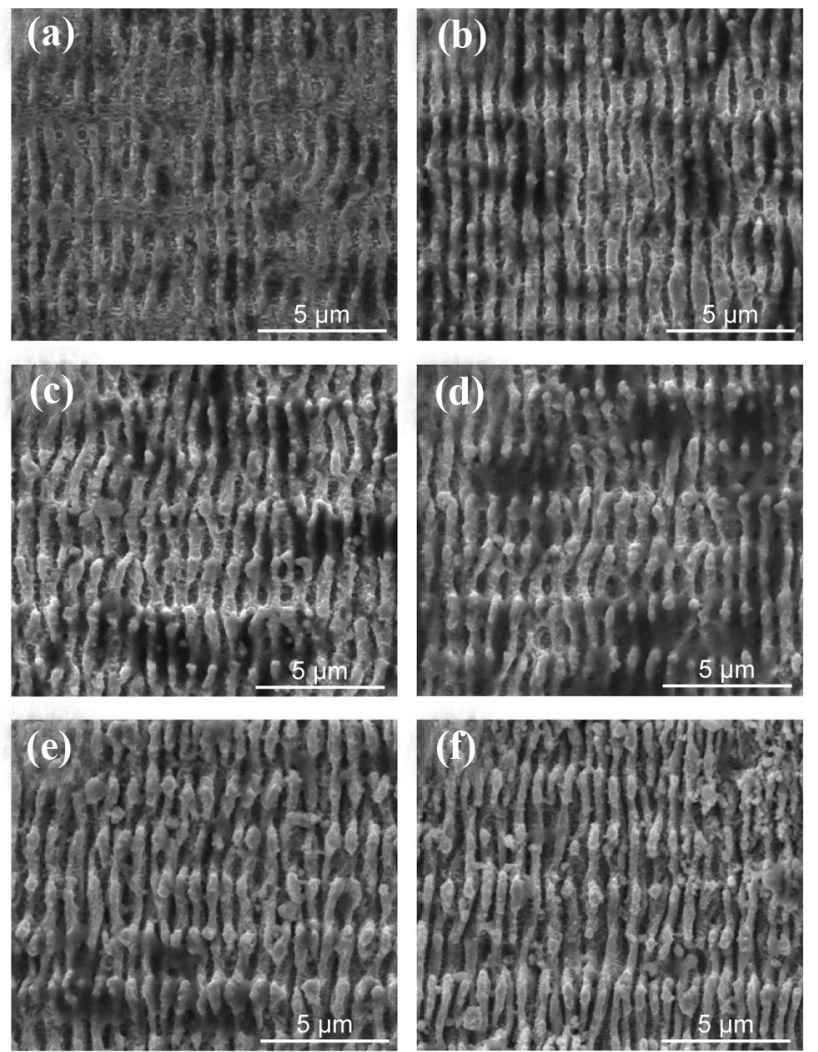

Fig. 4 SEM images of surfaces irradiated at fluence of $0.15 \mathrm{~J} / \mathrm{cm}^{2}$ and $N$ of a $1, \mathbf{b} 3, \mathbf{c} 5, \mathbf{d} 10$, e $50, \mathbf{f} 100$

\subsection{Cross-Scanning for Local Rewriting of LIPSS}

In this section, the laser was cross-scanned in perpendicular directions as shown in Fig. 2, and the possibility local rewriting of LIPSS morphology was investigated. For both the first and the second irradiations, single scan was performed $\left(N_{1}=N_{2}=1\right)$ at fluence of $0.15 \mathrm{~J} / \mathrm{cm}^{2}$ and a scanning speed of $40 \mathrm{~mm} / \mathrm{s}$. In the first irradiation, the scan pitch was set to $2 \mu \mathrm{m}$, while in the second irradiation, the scan pitch was set to 2 and $6 \mu \mathrm{m}$, respectively.

Figure 5 presents the SEM images of the irradiated surfaces. When the scan pitch in the second irradiation is $2 \mu \mathrm{m}$, the LIPSS generated in the first irradiation is rewritten and LIPSS perpendicular to the polarization of the second irradiation is newly created (Fig. 5a, b). When the scan pitch is $6 \mu \mathrm{m}$, however, the LIPSS formed in the first irradiation is partially rewritten. As a result, two kinds of LIPSS locally perpendicular to each other are generated on the same surface (Fig. 5c, d). Furthermore, as seen from Fig. $5 \mathrm{~b}$ and d, in the boundary region, the two kinds of LIPSS are partially connected with each other. 

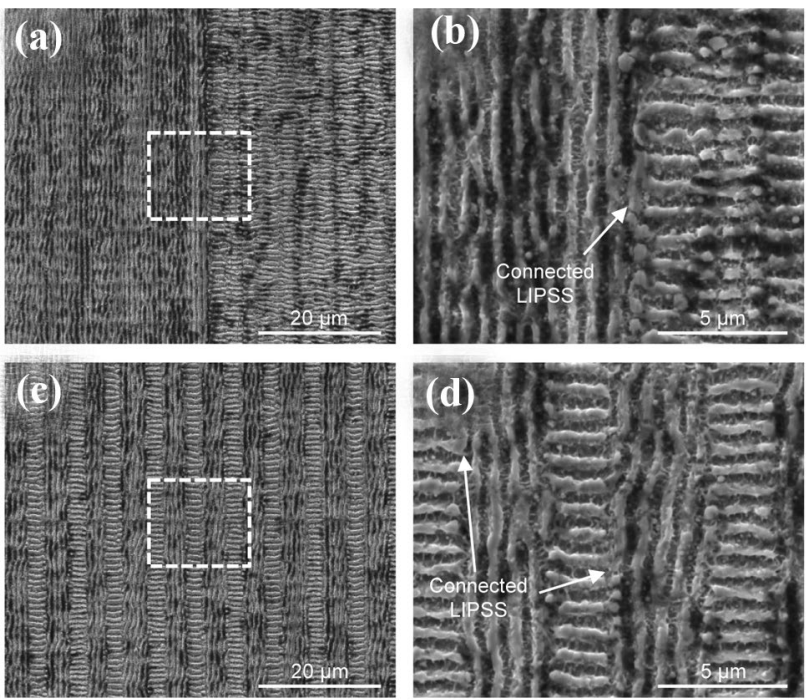

Fig. 5 SEM images of surfaces irradiated with crossing scans: a scan pitch $2 \mu \mathrm{m} ; \mathbf{b}$ close-up of $\mathbf{a} ; \mathbf{c}$ scan pitch $6 \mu \mathrm{m} ; \mathbf{d}$ close-up of $\mathbf{c}$

\subsection{Cross Scanning for Generating Nanodot Structure}

To find conditions for generating nanodot structures, the effects of number of scans $N_{1}$ and $N_{2}$ on the morphology of LIPSS in cross-scan irradiation tests were investigated. For both the first and second irradiations, the laser was irradiated at fluence of $0.15 \mathrm{~J} / \mathrm{cm}^{2}$, and a scanning speed of $40 \mathrm{~mm} / \mathrm{s}$ and a scan pitch of $2 \mu \mathrm{m}$. The number of scans $N_{1}$ was changed from 1 to 5 , and $N_{2}$ was set to 1 , respectively. Figure 6 presents the SEM images of the irradiated surfaces. When $N_{1}=N_{2}=1$, the LIPSS generated in the first irradiation is completely rewritten by the new LIPSS generated in the second irradiation. When $N_{1}=3$ and $N_{2}=1$, the LIPSS obtained in the first irradiation (vertical line) is partially rewritten by the new LIPSS (horizontal line) in the second irradiation, and some vertical lines still remain. However, when $N_{1}=5$ and $N_{2}=1$, the LIPSS formed in the
Fig. 6 SEM images of the surfaces irradiated at fluence $0.15 \mathrm{~J} / \mathrm{cm}^{2}$, scanning speed $40 \mathrm{~mm} / \mathrm{s}$, and scan pitch $2 \mu \mathrm{m}$ with various number of scans for each irradiation

\begin{tabular}{|c|c|c|}
\hline & After first irradiation & After second irradiation \\
\hline $\begin{array}{l}N_{1}=1 \\
N_{2}=1\end{array}$ & 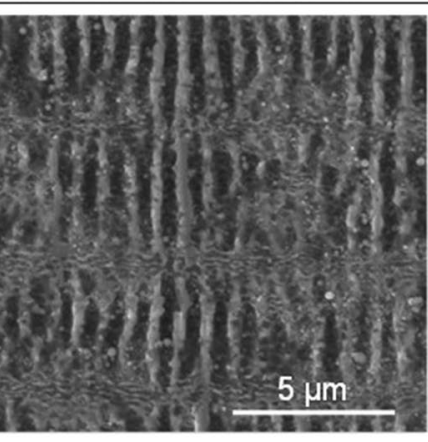 & 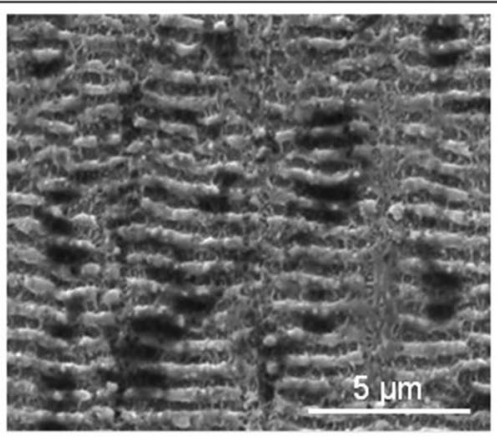 \\
\hline $\begin{array}{l}N_{1}=3 \\
N_{2}=1\end{array}$ & (19) & 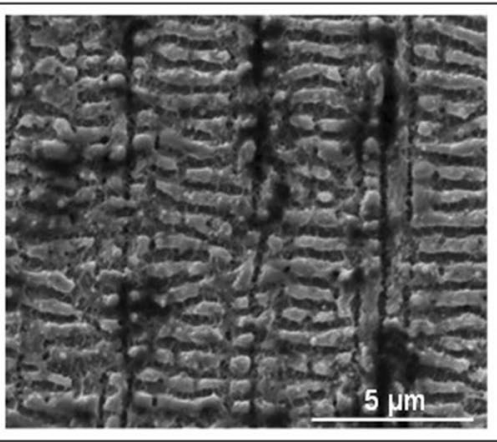 \\
\hline $\begin{array}{l}N_{1}=5 \\
N_{2}=1\end{array}$ & $15 \mu \mathrm{m}$ & 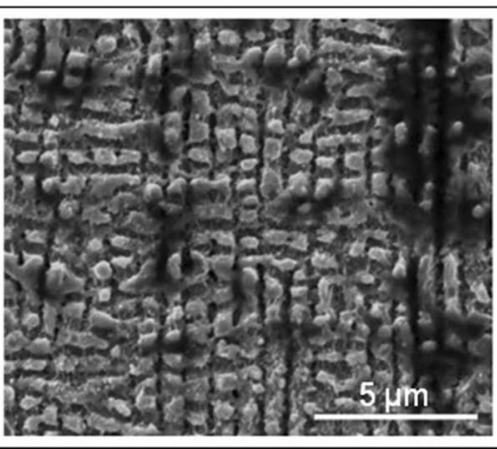 \\
\hline
\end{tabular}


first irradiation is changed to a dot structure after the second irradiation.

To investigate the formation mechanism of the dot structure, the LIPSS depth was measured using an AFM. The AFM images of the LIPSS formed in the first irradiation at different number of scans $\left(N_{1}=1,5\right)$ are shown in Fig. 7. The cross-sectional profiles of the LIPSS taken from the AFM image are also shown in the figure. It is clear that the depth of LIPSS increases as $N_{1}$ increases. When $N_{1}=1$, the average depth of LIPSS is $119 \mathrm{~nm}$; while the depth becomes $235 \mathrm{~nm}$ at $N_{1}=5$.

From this result, it may be said that the depth of LIPSS generated in the first irradiation affects the morphology of LIPSS in the second irradiation. That is to say, there is a historical effect in LIPSS formation, as shown in Fig. 8. When $N_{1}=1$, the LIPSS generated in the first irradiation is shallow, and can be completely restructured by the new LIPSS in the second irradiation. As a result, LIPSS perpendicular to the polarization of the second irradiation remains. From this result, it is presumable that the dominant mechanism of LIPSS formation for stainless steel is the laserinduced reorganization/reconstruction (without removal) of the near surface layer of the material, rather than the modulated ablation (with removal) induced by interference of laser and the SPP.

When $N_{1}=5$, however, the LIPSS formed in the first irradiation is so deep that it remains even after the second irradiation. As a result of orthogonal overlapping effect of the
Fig. 7 AFM images of LIPSS formed in the first irradiation when a $N_{1}=1$, b $N_{1}=5$. The cross-sectional profiles on the right side were taken along the dotted lines in the AFM images at the left side
Fig. 8 Schematic of laserinduced reorganization/reconstruction of top layer of material surface $\left(N_{1}=1\right)$ and historical effect of LIPSS in deep region and mechanism of nanodot structure formation $\left(N_{1}=5\right)$ (a)
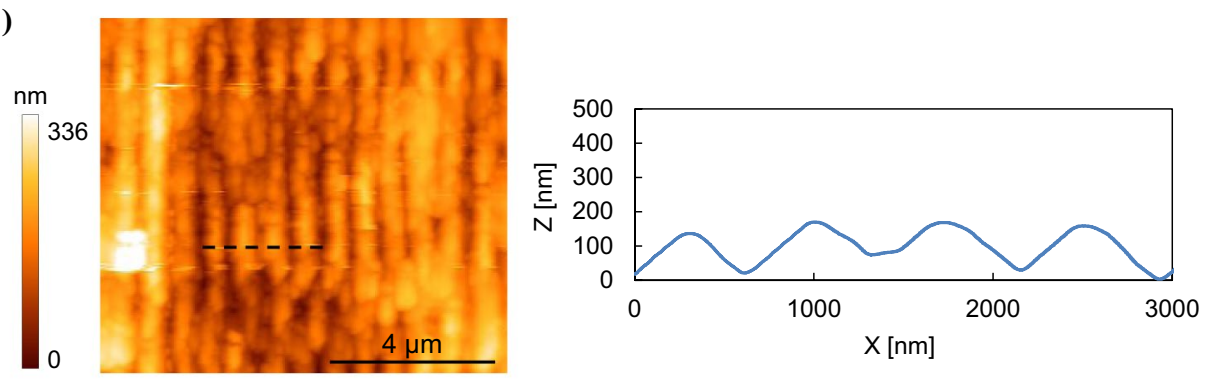

(b)
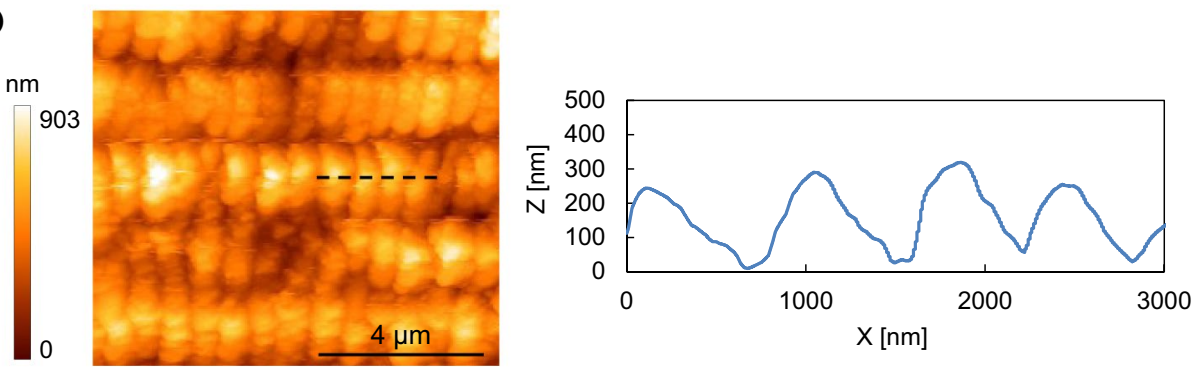

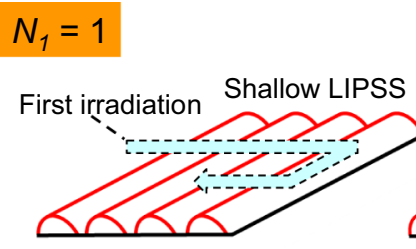

(a) First irradiation

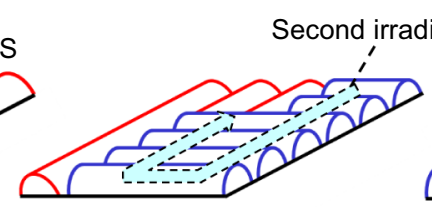

(b) Surface restructuring

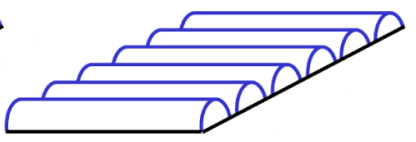

(c) New LIPSS

\section{$N_{1}=5$}

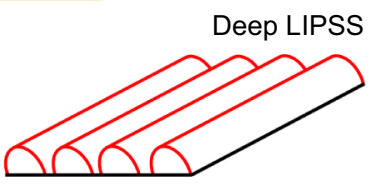

(a) First irradiation

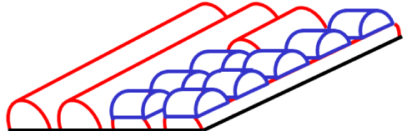

(b) Orthogonal LIPSS

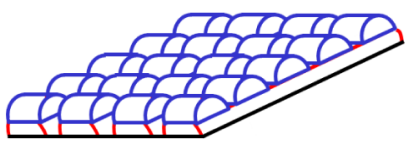

(c) Dot structure 
two kinds of LIPSS, the dot structure is created. This result indicates that the laser-induced reorganization/reconstruction phenomenon only occurs to the top layer of the material surface while the deeper region is less affected, i.e., there is a kind of historical effect. To achieve the nanodot structures, the number of laser scans in the first irradiation should be always more than that in the second irradiation.

\subsection{Surface Wettability Change}

The wettability of laser-irradiated surfaces with LIPSS and dot structure was investigated and compared with that of unirradiated surfaces. The SEM images of the sample surfaces and the corresponding results of contact angle measurements after laser irradiation as well as after exposure in the air for 7 days are shown in Fig. 9. The contact angle of water droplet is $81^{\circ}$ on the unirradiated surface, $20^{\circ}$ on the surface with LIPSS, and $10^{\circ}$ on the surface with nanodot structure. Super hydrophilicity is confirmed for the laserirradiated surfaces with nanodot structures. This result strongly demonstrates that LIPSS-based nanostructuring is effective for improving surface properties, such as wettability, of stainless-steel surfaces.

However, it should be pointed out that the contact angle is greatly changed after exposing the laser-irradiated samples in the air for 7 days (Fig. 9g-i). The contact angle of a water droplet on the nanostructured surface is greatly increased and it becomes larger than that of the unirradiated surface. A possible reason for this phenomenon is that an extremely thin layer of organic substance is adsorbed on the laser-irradiated metal surface after exposing in the air, and the surface becomes hydrophilic [28].

\section{Conclusions}

A stainless-steel surface was processed by a picosecond pulsed laser under various conditions. When increasing the number of laser scans, a transition from HSFL to LSFL occurred, and the depth of LIPSS increased. For single laser scans, the LIPSS that formed in the previous irradiation could be rewritten to LIPSS perpendicular to the
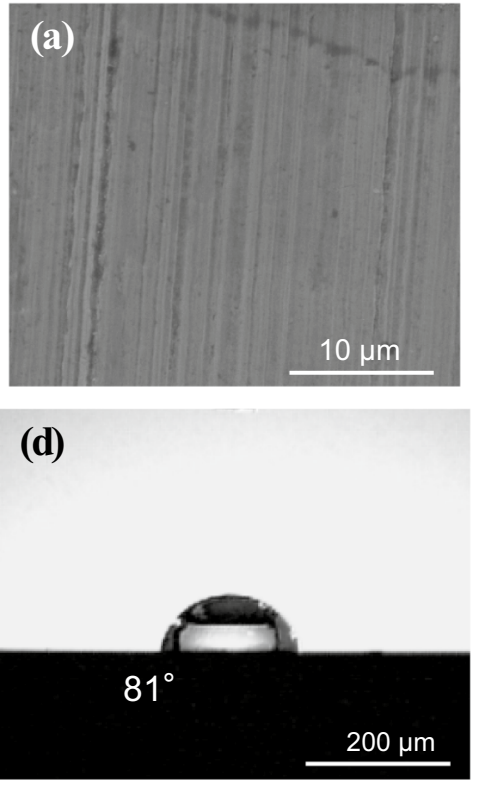

(g)

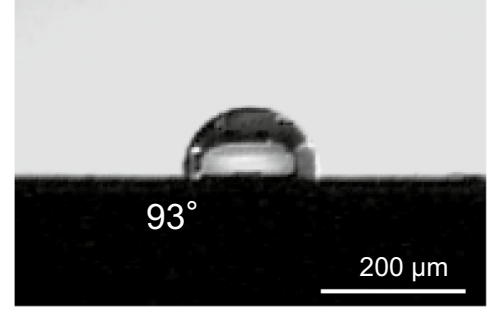

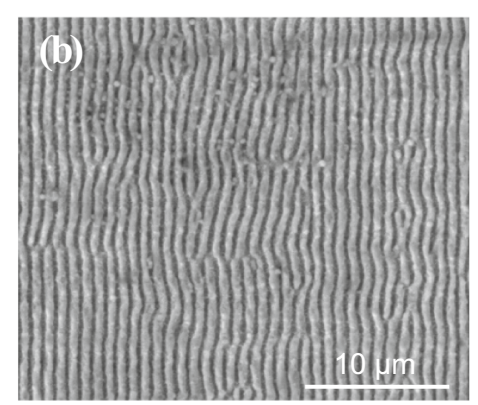

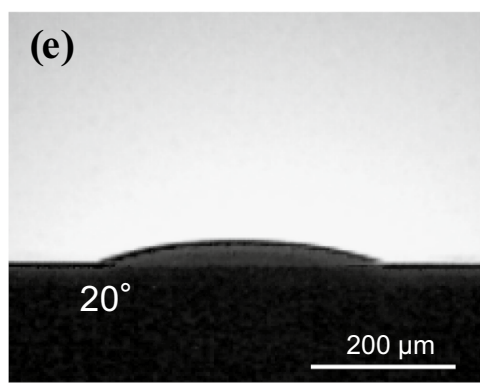

(h)

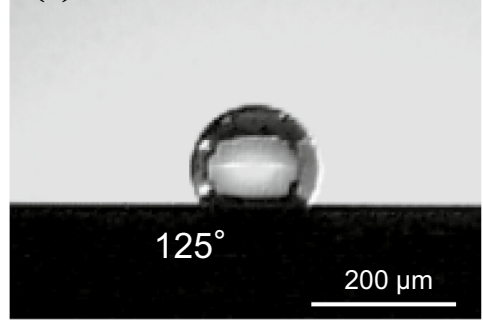

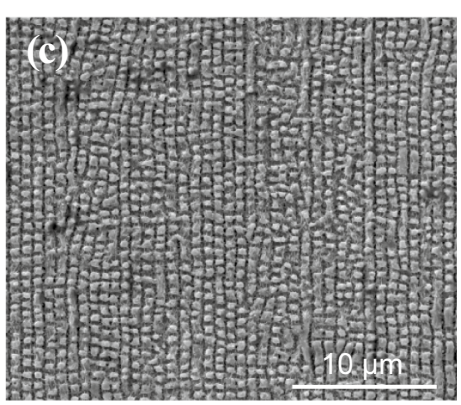

(f)

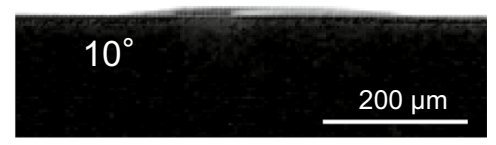

(i)

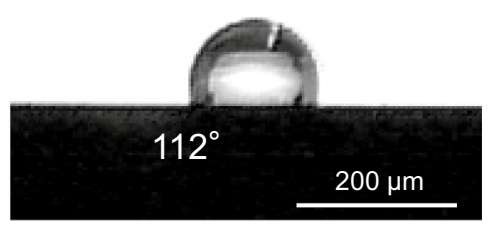

Fig. 9 SEM images of sample surfaces: a unirradiated, $\mathbf{b}$ with LIPSS, $\mathbf{c}$ with nanodot structure. $\mathbf{d}$, e, $\mathbf{f}$ are optical images of water droplets on surfaces $(\mathbf{a}, \mathbf{b}, \mathbf{c}) ; \mathbf{g}, \mathbf{h}, \mathbf{i}$ are results after exposure in the air for 7 days 
polarization of the subsequent irradiation. This enables local alteration of LIPSS directions. In contrast, deep LIPSS generated by multiple laser scans exhibited a kind of historical effect, i.e., LIPSS generated by the previous irradiation could not be rewritten, but remained after the subsequent irradiations. By applying this LIPSS formation mechanism to cross-scanning of the laser beam, nanodot structure was successfully created. Super hydrophilicity was confirmed for the irradiated surfaces with nanodot structures. The findings from this study demonstrate that LIPSS-based nanostructuring is an effective approach to improve or locally modify the surface properties of stainless-steel surfaces.

Open Access This article is licensed under a Creative Commons Attribution 4.0 International License, which permits use, sharing, adaptation, distribution and reproduction in any medium or format, as long as you give appropriate credit to the original author(s) and the source, provide a link to the Creative Commons licence, and indicate if changes were made. The images or other third party material in this article are included in the article's Creative Commons licence, unless indicated otherwise in a credit line to the material. If material is not included in the article's Creative Commons licence and your intended use is not permitted by statutory regulation or exceeds the permitted use, you will need to obtain permission directly from the copyright holder. To view a copy of this licence, visit http://creativecommons.org/licenses/by/4.0/.

\section{References}

1. Vorobyev AY, Makin VS, Guo C (2007) Periodic ordering of random surface nanostructures induced by femtosecond laser pulses on metals. J Appl Phys 101:034903

2. Kobayashi T, Sera H, Wakabayashi T, Endo H, Takushima Y, Yan J (2018) Surface flattening and nanostructuring of steel by picosecond pulsed laser irradiation. Nanomanuf Metrol 1:1-8

3. Tomita T, Kinoshita K, Matsuo S, Hashimoto S (2007) Effect of surface roughening on femtosecond laser-induced ripple structures. Appl Phys Lett 90:153115

4. Bonse J, Krüger J, Höhm S, Rosenfeld A (2012) Femtosecond laser-induced periodic surface structures. J Laser Appl 24:042006

5. Gregorčič P, Sedlaček M, Podgornik B, Reif J (2016) Formation of laser-induced periodic surface structures (LIPSS) on tool steel by multiple picosecond laser pulses of different polarizations. Appl Surf Sci 387:698-706

6. Vorobyev AY, Guo C (2007) Femtosecond laser structuring of titanium implants. Appl Surf Sci 253:7272-7280

7. Borowiec A, Haugen HK (2003) Subwavelength ripple formation on the surfaces of compound semiconductors irradiated with femtosecond laser pulses. Appl Phys Lett 82:4462

8. Wagner R, Gottmann J, Horn A, Kreutz EW (2006) Subwavelength ripple formation induced by tightly focused femtosecond laser radiation. Appl Surf Sci 252:8576-8579

9. Reif J, Varlamova O, Costache F (2008) Femtosecond laser induced nanostructure formation self-organization control parameters. Appl Phys A 92:1019-1024

10. Vorobyev AY, Guo C (2007) Effects of nanostructure-covered femtosecond laser-induced periodic surface structures on optical absorptance of metals. Appl Phys A 86:321-324

11. Sakabe S, Hashida M, Tokita S, Namba S, Okamura K (2009) Mechanism for self-formation of periodic grating structures on a metal surface by a femtosecond lase pulse. Phys Rev B 79:033409
12. Bonse J, Rosenfeld A, Krüger J (2009) On the role of surface plasmon polaritons in the formation of laser-induced periodic surface structures upon irradiation of silicon by femtosecond-laser pulses. J Appl Phys 106:104910

13. Varlamova O, Costache F, Reif J, Bestehorn M (2006) Selforganized pattern formation upon femtosecond laser ablation by circularly polarized light. Appl Surf Sci 252:4702-4706

14. Kobayashi T, Wakabayashi T, Takushima Y, Yan J (2019) Formation behavior of laser-induced periodic surface structures on stainless tool steel in various media. Precision Engineering $57: 244-252$

15. Wu B, Zhou M, Li J, Ye X, Li G, Cai L (2009) Superhydrophobic surfaces fabricated by microstructuring of stainless steel using femtosecond laser. Appl Surf Sci 256:61-66

16. Calderon MM, Rodríguez A, Ponte AD, Miñana MCM, Aranzadi MG, Olaizola SM (2016) Femtosecond laser fabrication of highly hydrophobic stainless steel surface with hierarchical structures fabricated by combining ordered microstructures and LIPSS. Appl Surf Sci 374:81-89

17. Bonse J, Koter R, Hartelt M, Spaltmanm D, Pentzien S, Höhm S, Rosenfeld A, Krüger J (2014) Femtosecond laser-induced periodic surface structures on steel and titanium alloy for tribological applications. Appl Phys A 117:103-110

18. Yao J, Zhang C, Liu H, Dai Q, Wu L, Lan S, Gopal AV, Trofimov VA, Lysak TM (2012) Selective appearance of several laserinduced periodic surface structure patterns on a metal surface using structural colors produced by femtosecond laser pulses. Appl Surf Sci 258:7625-7632

19. Vorobyev AY, Guo C (2008) Colorizing metals with femtosecond laser pulses. Appl Phys Lett 92:041914

20. Vorobyev AY, Guo C (2008) Femtosecond laser-induced periodic surface structure formation on tungsten. J Appl Phys 104:063523

21. Bonse J, Höhm S, Rosenfeld A, Krüger J (2013) Sub-100-nm laser-induced periodic surface structures upon irradiation of titanium by Ti sapphire femtosecond laser pulses in air. Appl Phys A 110:547-551

22. Qi L, Nihii K, Namba Y (2009) Regular subwavelength surface structures induced by femtosecond laser pulses on stainless steel. Opt Lett 34:12

23. Bonse J, Krüger J (2010) Pulse number dependence of laserinduced periodic surface structures for femtosecond laser irradiation of silicon. J Appl Phys 108:034903

24. Hwang TY, Guo C (2010) Angular effects of nanostructure-covered femtosecond laser induced periodic surface structures on metals. J Appl Phys 108:073523

25. Dufft D, Rosenfeld A, Das SK, Grunwald R, Bonse J (2009) Femtosecond laser-induced periodic surface structures revisited: a comparative study on ZnO. J Appl Phys 105:034908

26. Ling EJY, Saïd J, Brodusch N, Gauvin R, Servio P, Kietrig AM (2015) Investigating and understanding the effects of multiple femtosecond laser scans on the surface topography of stainless steel 304 and titanium. Appl Surf Sci 353:512-521

27. Yao JW, Zhang CY, Liu HY, Dai QF, Wu LJ, Lan S, Gopal AV, Trofimov VA, Lysak TM (2012) High spatial frequency periodic structures induced on metal surface by femtosecond laser pulses. Opt Express 20:905-911

28. Zhang Y, Zou G, Liu L, Zhao Y, Liang Q, Wu A, Zhou YN (2016) time dependent wettability of nano patterned surfaces fabricated by femtosecond laser with high efficiency. Appl Surf Sci 389:554-559 